\title{
Optimal impact isolation for injury prevention evaluated by the head injury criterion
}

\author{
D.V. Balandin ${ }^{\mathrm{a}}$, N.N. Bolotnik ${ }^{\mathrm{b}}$, J.R. Crandall ${ }^{\mathrm{c}}$, W.D. Pilkeyc,* and S.V. Purtsezov ${ }^{\mathrm{c}}$ \\ ${ }^{a}$ Department of Computational Mathematics and Cybernetics, Nizhny Novgorod State University, 23 Gagarin Ave., \\ Nizhny Novgorod 603950, Russia \\ ${ }^{\mathrm{b}}$ Institute for Problems in Mechanics of Russian Academy of Sciences, 101-1 Prospect Vernadskogo, Moscow \\ 119526, Russia \\ ${ }^{\mathrm{c}}$ Department of Mechanical and Aerospace Engineering, University of Virginia, 122 Engineer's Way, P.O. Box \\ 400746, Charlottesville, VA 22904-4746, USA
}

Received 14 July 2006

\begin{abstract}
The optimal control of the deceleration of a particle moving along a straight line after an impact against an isolated surface is considered. The force applied to the particle by the surface is treated as the control variable. The deceleration distance is minimized subject to a constraint on the Head Injury Criterion functional. This functional is an integral criterion that is utilized in engineering biomechanics to evaluate the expected severity of impact-induced head injury of a human being. The solution obtained provides characteristics of the limiting capabilities for the prevention of head injuries by means of an impact isolator, such as a coating of the surface against which the impacts occur. The head injuries can be due to impact occurrences, including traffic crashes, falling, and contacts with ballistic objects.
\end{abstract}

Keywords: Shock isolation, optimal control, head injury criterion

\section{Introduction}

The Head Injury Criterion (HIC) is an empirical integral criterion that evaluates the possible severity of human brain injury induced by an impact in terms of kinematic parameters of this impact. Formally, this criterion is defined by

$$
\mathrm{HIC}=\max _{t_{1}, t_{2}, t_{2}-t_{1} \leqslant \Delta}\left\{\left[\frac{1}{t_{2}-t_{1}} \int_{t_{1}}^{t_{2}} a(t) d t\right]^{2.5}\left(t_{2}-t_{1}\right)\right\},
$$

where $a(t)$ is the magnitude of the acceleration of the center of mass of the brain, and $\Delta$ is a constant parameter having the dimension of time. The criterion of Eq. (1) reflects the dependence of the severity of the injury on both the mean magnitude of the head acceleration induced by the impact pulse and the duration of this pulse. With the increase of either or both of these quantities the expected severity of the head injury increases. In Eq. (1), the expression in the square brackets is the head acceleration mean magnitude on the time interval $t_{1} \leqslant t \leqslant t_{2}$.

The HIC functional is utilized as a standard crashworthiness criterion for transport vehicles (road and airborne) and as the basic quality index for playground (sporting and children) surfaces and helmets. To establish the degree of correspondence to the standard, the vehicle, structure, or equipment should be subjected to tests the conditions of which are specified in appropriate documents. Typically, the equipment for such tests involves a human dummy

\footnotetext{
*Corresponding author. E-mail: wdp@virginia.edu.
} 
with sensors to measure the parameters of the motion occurring in the functionals regarded as the injury criteria. The HIC tests require three accelerometers to be placed at the center of mass of the dummy's head to measure three components of the acceleration of that point.

The definition of the HIC functional implies that its dimension is $L^{2.5} / T^{4}$, where $L$ is the dimension of the unit of length and $T$ is the dimension of the unit of time. For example, in SI units HIC is measured in $m^{2.5} / s^{4}$. In the literature on impact biomechanics and crashworthiness, the acceleration in the expression for HIC is conventionally measured in units of the mean value of the acceleration due to gravity on the earth surface $\left(g \approx 9.81 \mathrm{~m} / \mathrm{s}^{2}\right)$ and time in seconds. In this case, as a rule, only the numerical value of this functional is given and the dimension (seconds) is omitted.

The value of the HIC (for a prescribed function $a(t)$ ) depends on the parameter $\Delta$. This parameter bounds the duration of the time intervals $t_{1} \leqslant t \leqslant t_{2}$ for which the interval providing the maximum for the expression in the curly brackets in Eq. (1) is calculated. The value of $\Delta$ is specified by test standards. For example, car crash test standards specify $\Delta=15 \mathrm{~ms}$ or $\Delta=36 \mathrm{~ms}$. Note that the value of HIC does not decrease as $\Delta$ increases, since in this case, the class of time intervals with respect to which the maximization in Eq. (1) is performed increases.

We choose to provide a brief review of development of the HIC functional and its biomechanical validation. The HIC is based on biomechanical experiments aimed at the investigation of the influence of the magnitude and duration of the acceleration pulse occurring on the characteristics of brain injury. Gurdjian and his colleagues [5-7] at the Wayne State University (Michigan, USA) were the first to perform such investigations in the 1950s. Laboratory animals (dogs and monkeys) and human cadavers were utilized for the experiments. The head was subjected to an impact loading. The duration of the impact pulse, the average acceleration of a certain reference point of the head over that duration, as well as the intracranial pressure were measured. The intracranial pressure indicated the degree of severity of the head injury. The distinction was made between major (severe) injuries, which were associated with irreversible changes that lead to serious malfunctions of the brain or death, and minor (less severe) injuries in which such changes were not observed. The experimental results were processed as follows. Each experiment was marked by a point on the coordinate plane $(\Delta t, \bar{a})$, where $\Delta t$ is the duration of the impact pulse and $\bar{a}$ is the mean acceleration of the reference point of the head over this time, time being measured in seconds and the acceleration in $g$. For each point, the degree of severity of the head injury (major or minor) was indicated and the curve that separated the points corresponding to major injuries from those corresponding to minor injuries was plotted. This curve (Fig. 1) is known as the Wayne State University Cerebral Concussion Tolerance Curve (WSTC). When plotted on the logarithmic scale (i.e., in the $\log \Delta t-\log \bar{a}$ coordinates), the tolerance curve is close to a straight line. The least squares linear approximation of this curve on the logarithmic scale yields

$$
2.5 \log \bar{a}+\log \Delta t=3,
$$

where $\log$ denotes the logarithm to the base 10. The relation of Eq. (2) can be rewritten in the form

$$
\bar{a}^{2.5} \Delta t=1000 \text {. }
$$

Gadd [4] and Versace [13] proposed quantitative integral measures for head injury severity on the basis of the left-hand side of Eq. (3). Gadd's measure (Gadd severity index) is defined by the integral

$$
G=\int_{t_{1}}^{t_{2}}[a(t)]^{2.5} d t,
$$

where $t_{1}$ and $t_{2}$ are the starting and end instants of the impact pulse, respectively, and $a$ is the magnitude of the acceleration of the brain center of mass.

Versace's measure is defined by the expression

$$
V=\left[\frac{1}{t_{2}-t_{1}} \int_{t_{1}}^{t_{2}} a(t) d t\right]^{2.5}\left(t_{2}-t_{1}\right) .
$$

This measure coincides with the left-hand side of relation Eq. (3), since the duration of the impact pulse, $\Delta t$, is defined by the difference $t_{2}-t_{1}$, and the expression in the square brackets is the mean acceleration over this time 


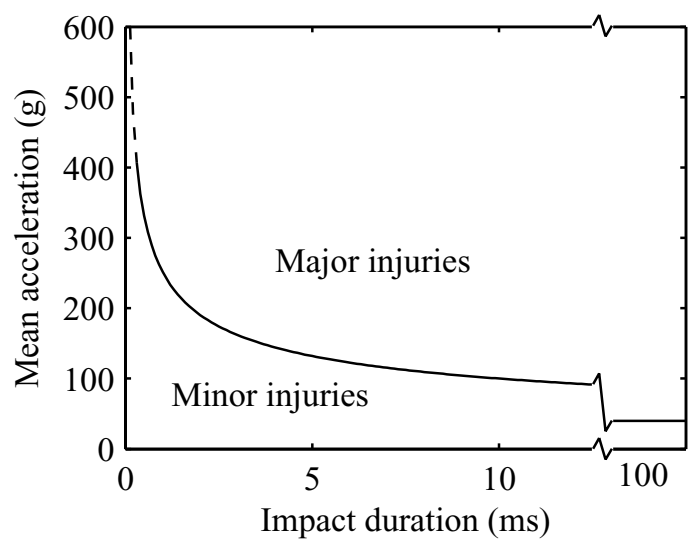

Fig. 1. Wayne State University Cerebral Concussion Tolerance Curve.

interval. On the other hand, the functional $V$ coincides in form with the expression to be maximized in the HIC functional of Eq. (1). For both measures of Eqs (4) and (5), the value 1000 is regarded as critical; exceeding this value threatens one with severe head injuries.

Unlike the criteria of Eqs (4) and (5), in the criterion of Eq. (1), the time interval on which the expression in the curly brackets is calculated is to be chosen on the basis of maximization, rather than being fixed. This is done to avoid obtaining an underestimated value if the impact pulse involves time intervals on which the acceleration magnitude is substantially less than its maximum value.

Although the HIC is utilized in a number of countries as a standard crashworthiness criterion of transport vehicles and some structures (for example, sporting playgrounds [12]) with respect to head injuries and as the basic performance index for impact protection helmets, the biomechanical adequacy of this measure is still being disputed and was criticized in a number of papers (see, e.g., $[9,10])$.

We choose to use HIC as a tolerance criterion to be taken into account when designing systems for which impacts of the head against various surfaces can occur. These surfaces should be covered with impact isolation materials. The mechanical properties of these materials and the thickness of the coating should be chosen so as to provide HIC values lying below the major injury threshold for impacts with typical velocities. (The range of these velocities is usually defined by appropriate standards.)

One of the basic problems associated with the creation of impact isolation coatings is that of determining the minimal thickness of the coating that guarantees a prescribed degree of head injury prevention (in terms of the HIC). It is also of interest to know the specific features of the deceleration of the head by this coating. Furthermore, the inverse problem, in which the HIC functional is to be minimized provided that the thickness of the coating is constrained is important. From the mathematics point of view, such problems are stated as optimal control problems in which HIC is either to be minimized or constrained.

To the authors' knowledge, a comprehensive analysis of the optimal control problems with the HIC functional has not yet been performed, although an optimal control has been constructed in some special cases. For example, the solution is known for the problem of the optimal deceleration of a point mass (particle) moving along a straight line with a given initial velocity. The deceleration distance is minimized under the condition that the HIC does not exceed a prescribed admissible value, with the parameter $\Delta$ being assumed to be equal to the deceleration pulse duration. This model allows one to analyze the limiting capabilities of the isolation of the head from impacts for helmets or coatings of surfaces against which the impacts can occur. The analytical solution of this problem is presented (without proof) in [11] in connection with the crashworthy design of the car hood to reduce the head injury of a pedestrian caused by an impact against the hood. In [3], a numerical solution of a similar problem was constructed in connection with the limiting performance analysis of impact protection helmets.

In [8], mathematical features of the HIC functional are studied for the case where the function $a(t)$ in Eq. (1) is defined on a finite time interval, vanishes at the ends of this interval, is continuous and piecewise differentiable, and the parameter $\Delta$ coincides with the length of the interval on which the function $a(t)$ is defined. The class of functions 
$a(t)$ considered in [8] covers all cases that can be encountered in practice. Therefore, the algorithms proposed in the cited paper are suitable for the determination of the upper bound for the HIC (i.e., the maximum of the functional of Eq. (1) over all delta $\Delta$ ) when processing crash-test measurement data. At the same time, for theoretical analyses of problems involving the HIC, in particular, optimal control problems, it is reasonable to extend the class of functions $a(t)$ to that of functions integrable (generally speaking, in the improper sense) on a prescribed time interval. In what follows, we will show that the solution of optimal control problems with the HIC functional can lead to functions $a(t)$ tending to infinity at some time instants, the number of such instants depending on the parameter $\Delta$.

In the present paper, a complete solution is given for the problem of the optimal deceleration of the rectilinear motion of a particle. The particle is acted upon by the control force that models the interaction with the surface against which the impact has occurred. The deceleration distance is minimized subject to a constraint on the HIC functional. In contrast to [3,11], the parameters of the problem - the mass of the particle, the initial velocity (impact velocity), the maximum allowable value of the HIC, and the parameter $\Delta$ occurring in the definition of this criterion are arbitrary. Analytical expressions are obtained for the optimal control and the minimal deceleration distance. The qualitative behavior of the optimal control is investigated as a function of these parameters. The optimal control is compared with other control laws in terms of the deceleration distance.

\section{Statement of the optimal control problem}

Consider a particle of mass $m$ moving along a straight line under the action of the force $u$. Such a motion is governed by the differential equation

$$
m \ddot{x}=u,
$$

subjected to the initial conditions

$$
x(0)=0, \dot{x}(0)=v_{0},
$$

where $x$ is the coordinate of the particle measured from some initial position in a fixed (inertial) reference frame and $v_{0}$ is the initial velocity. Without loss of generality we assume $v_{0}>0$.

The system of Eqs (6) and (7) can be utilized as a model of the motion of the center of mass of a body after an impact against a surface, if the velocity of the body at the impact instant is directed along the normal to the surface. In this case, $u$ is the force of interaction of the body with the impact surface, and $v_{0}$ is the velocity of the body center of mass at the instant of the impact. The positive values of the coordinate $x$ measure the deformation of the surface.

Also, this model describes the motion of the center of mass of a body placed in an impact isolation container after the latter has come to an instantaneous stop as a result of a perfectly inelastic impact against a rigid surface. The body to be protected from the impact is separated from the container walls by a relatively soft padding that deforms thereby reducing the impact force. In this case, $u$ is the force exerted on the body by the padding and $x$ measures the deformation of the padding.

The model of Eqs (6) and (7) can be utilized for the analysis of the limiting capabilities of protection of the head from impacts by means of impact isolation coatings or helmets, if the severity of the head injury is measured by HIC, since this criterion takes into account only the motion of the center of mass of the head. Of course, when hitting a surface, the head interacts not only with the coating of this surface or with the padding of the helmet but also with the remaining portion of the human body. However, when considering the motion of the head on the time interval the duration of which is close to that of impact (defined, for example, as the time of the deceleration of the head center of mass in coming to a complete stop due to the deformed coating or padding), one can neglect this interaction for many types of impact in view of the relatively high stiffness of the coating or padding, as compared, for example, with the bending rigidity of the cervical spine.

We will characterize the quality of the impact isolation in terms of the model under consideration by the peak magnitude of the displacement of the particle,

$$
J_{1}=\max _{t \in[0, \infty)}|x(t)|,
$$

and the HIC functional, 


$$
J_{2}=\max _{t_{1}, t_{2}, t_{2}-t_{1} \leqslant \Delta}\left\{\left[\frac{1}{t_{2}-t_{1}} \int_{t_{1}}^{t_{2}} \frac{|u(t)|}{m} d t\right]^{2.5}\left(t_{2}-t_{1}\right)\right\} .
$$

Consider the optimal control problem for the system of Eqs (6) and (7).

Problem 1. For the system governed by the differential equation of Eq. (6) subjected to the initial conditions of Eq. (7), find an optimal control $u=u_{0}(t)$ in the class of integrable functions that minimizes the peak displacement $J_{1}$, provided that the criterion $J_{2}$ does not exceed the prescribed positive number $H$, i.e.,

$$
J_{1}\left(u_{0}\right)=\min _{u}\left\{J_{1}(u) \mid J_{2}(u) \leqslant H\right\} .
$$

This problem is characterized by 4 parameters $-m, v_{0}, \Delta$, and $H$. The transition to the dimensionless (primed) variables

$$
\begin{aligned}
& x^{\prime}=\frac{H^{2 / 3}}{v_{0}^{8 / 3}} x, t^{\prime}=\frac{H^{2 / 3}}{v_{0}^{5 / 3}} t, u^{\prime}=\frac{u}{m}\left(\frac{v_{0}}{H}\right)^{2 / 3}, \Delta^{\prime}=\frac{H^{2 / 3}}{v_{0}^{5 / 3}} \Delta, \\
& J_{1}^{\prime}=\frac{H^{2 / 3}}{v_{0}^{8 / 3}} J_{1}, J_{2}^{\prime}=\frac{1}{H} J_{2}
\end{aligned}
$$

reduces this number to one. By performing the change of variables of Eq. (11) in the relations of Eqs (6)-(10) and omitting the primes, we obtain the relations of the same form but with $m=1, v_{0}=1$, and $H=1$. The only free parameter remaining after this change of variables is the dimensionless parameter $\Delta^{\prime}$.

When constructing the solution of the problem, we will utilize the dimensionless variables. The primes will be omitted, apart from the cases where the dimensionless variables are considered along with the dimensional ones.

\section{Construction of the solution}

To determine the minimum of the criterion $J_{1}$, it suffices to solve the problem stated in the previous section on the time interval $[0, T]$, where $T$ is the instant (unknown in advance) at which the velocity $\dot{x}(t)$ vanishes for the first time. For the control defined as $u(t) \equiv 0$ for $t>T$, the particle remains indefinitely at the position $x(T)$.

To construct the solution, we will perform a number of transformations. Integrate the equation of (6) subjected to the initial condition of Eq. (7) to obtain the expression for the velocity

$$
\dot{x}=1-v(t), v(t)=-\int_{0}^{t} u(\tau) d \tau .
$$

The constraint $J_{2} \leqslant 1$, where $J_{2}$ is defined by Eq. (9) with $m=1$, is equivalent to the inequality

$$
\left[\frac{1}{t_{2}-t_{1}} \int_{t_{1}}^{t_{2}}|u(\tau)| d \tau\right]^{5 / 2}\left(t_{2}-t_{1}\right) \leqslant 1,0<t_{2}-t_{1} \leqslant \Delta .
$$

Raise this inequality to a power of $2 / 5$ to represent it in the form

$$
\int_{t_{1}}^{t_{2}}|u(\tau)| d \tau \leqslant\left(t_{2}-t_{1}\right)^{3 / 5}, 0<t_{2}-t_{1} \leqslant \Delta .
$$

The last inequality implies

$$
-\int_{t_{1}}^{t_{2}} u(\tau) d \tau \leqslant\left(t_{2}-t_{1}\right)^{3 / 5}, 0<t_{2}-t_{1} \leqslant \Delta
$$


or, with reference to Eq. (12),

$$
v\left(t_{2}\right)-v\left(t_{1}\right) \leqslant\left(t_{2}-t_{1}\right)^{3 / 5}, 0<t_{2}-t_{1} \leqslant \Delta .
$$

Consider first the simple special case where $\Delta=\infty$. By setting $t_{1}=0$ and $t_{2}=t$ in Eq. (16) we obtain

$$
v(t) \leqslant t^{3 / 5} \text {. }
$$

The relations of Eqs (17) and (12) lead to the inequality

$$
\dot{x}(t) \geqslant 1-t^{3 / 5} \text {. }
$$

The integration of this inequality from 0 to $t$ for the initial condition $x(0)=0$ yields

$$
x(t) \geqslant t-\frac{5}{8} t^{8 / 5} .
$$

Hence, the lower bound of $x$ for any $t$ is defined by

$$
x(t)=t-\frac{5}{8} t^{8 / 5} .
$$

and is attained at $v(t)=t^{3 / 5}$, which, in accordance with Eq. (12), implies $u(t)=-(3 / 5) t^{-2 / 5}$. The variable $\dot{x}(t)$ vanishes at $t=1$. At this time instant, the variable $x(t)$ attains its maximum $x(1)=3 / 8$. Therefore, the control

$$
u(t)= \begin{cases}-\frac{3}{5} t^{-2 / 5}, & \text { if } t \leqslant 1 \\ 0, & \text { if } t>1\end{cases}
$$

is optimal in the case of $\Delta=\infty$, if this control satisfies the inequality of Eq. (16). We will prove this inequality.

For the control of Eq. (21), we have

$$
v(t)= \begin{cases}t^{3 / 5}, & \text { if } t \leqslant 1 \\ 1, & \text { if } t>1\end{cases}
$$

For $t_{2} \leqslant 1$, the inequality of Eq. (16) becomes

$$
t_{2}^{3 / 5}-t_{1}^{3 / 5}-\left(t_{2}-t_{1}\right)^{3 / 5} \leqslant 0, t_{2}>t_{1} .
$$

The relation of Eq. (23) can be represented as

$$
t_{2}^{3 / 5} \Psi(\xi) \leqslant 0, \Psi(\xi)=1-\xi^{3 / 5}-(1-\xi)^{3 / 5}, \xi=t_{1} / t_{2}, 0<\xi<1 .
$$

The function $\Psi(\xi)$ is continuous on the interval $[0,1]$, vanishes at the end points of this interval, is differentiable on the interval $(0,1)$ and convex downward $\left(\Psi^{\prime \prime}(\xi)>0\right.$ for $\left.0<\xi<1\right)$. Hence, $\Psi(\xi)<0$ for $0<\xi<1$, which proves the inequality of Eq. (24) and thereby Eq. (23).

Note that the inequality of Eq. (23) is valid for any $t_{1}$ and $t_{2}$ satisfying the condition $t_{2}>t_{1} \geqslant 0$, rather than only for $t_{2}<1$.

If $t_{1}<1$ and $t_{2} \geqslant 1$, the inequality of Eq. (16) for $v(t)$ defined by Eq. (22) can be represented in the form

$$
1-t_{1}^{3 / 5}-\left(t_{2}-t_{1}\right)^{3 / 5} \leqslant 0, t_{2}>t_{1} .
$$

To prove this inequality, it suffices to add the nonpositive quantity $1-t_{2}^{3 / 5}$ to both parts of Eq. (23).

For $t_{1}>1$ and $v(t)$ defined by Eq. (22), the left-hand side of Eq. (16) vanishes, whereas the right-hand side is positive and, hence, this inequality holds.

Thus, the control of Eq. (21) is optimal in the case of $\Delta=\infty$, with $J_{1}=3 / 8$ and $J_{2}=1$. This control remains optimal for any $\Delta \geqslant 1$. The proof of this fact is quite similar to the proof of the optimality in the case of $\Delta=\infty$.

Consider now the case of $\Delta<1$. To construct the optimal control we will follow the scheme that was utilized for the case of $\Delta=\infty$. First we will construct a lower bound for the variable $x(t)$, similar to that of Eq. (19), then find a control at which this lower bound is attained and verify that the constraint of Eq. (16) holds for this control.

Let $k \Delta<t \leqslant(k+1) \Delta$, where $k$ is a nonnegative integer. Set $t_{1}=k \Delta$ and $t_{2}=t$ in Eq. (16) to obtain 


$$
v(t) \leqslant v(k \Delta)+(t-k \Delta)^{3 / 5}, k \Delta<t \leqslant(k+1) \Delta, k=0,1,2, \ldots .
$$

For $t=(k+1) \Delta$, we have

$$
v((k+1) \Delta) \leqslant v(k \Delta)+\Delta^{3 / 5}, k=0,1,2, \ldots .
$$

With reference to the initial condition $v(0)=0$, from Eq. (27) it follows that $v(k \Delta) \leqslant k \Delta^{3 / 5}$. Substitute this upper bound for $v(k \Delta)$ into Eq. (26) to obtain

$$
v(t) \leqslant k \Delta^{3 / 5}+(t-k \Delta)^{3 / 5}, k \Delta<t \leqslant(k+1) \Delta, k=0,1,2, \ldots .
$$

The relations of Eqs (12) and (28) imply the lower bound for the velocity

$$
\dot{x}(t) \geqslant 1-k \Delta^{3 / 5}-(t-k \Delta)^{3 / 5}, k \Delta<t \leqslant(k+1) \Delta, k=0,1,2, \ldots .
$$

The integration of this inequality from 0 to $t$ with reference to the initial condition $x(0)=0$ yields the lower bound for the coordinate

$$
\begin{aligned}
x(t) \geqslant & k \Delta\left(1-\left(\frac{1}{8}+\frac{k}{2}\right) \Delta^{3 / 5}\right)+(t-k \Delta)\left(1-k \Delta^{3 / 5}\right)-\frac{5}{8}(t-k \Delta)^{8 / 5}, \\
& k \Delta<t \leqslant(k+1) \Delta .
\end{aligned}
$$

The inequalities of Eqs (29) and (30) are analogues of the inequalities of Eqs (18) and (19) for the case under consideration. From these inequalities it follows that the lower bound of the variable $x(t)$ for any $t$ is defined by

$$
\begin{aligned}
x(t)= & k \Delta\left(1-\left(\frac{1}{8}+\frac{k}{2}\right) \Delta^{3 / 5}\right)+(t-k \Delta)\left(1-k \Delta^{3 / 5}\right)-\frac{5}{8}(t-k \Delta)^{8 / 5}, \\
& k \Delta<t \leqslant(k+1) \Delta
\end{aligned}
$$

and is attained at

$$
v(t)=k \Delta^{3 / 5}+(t-k \Delta)^{3 / 5}, k \Delta<t \leqslant(k+1) \Delta, k=0,1,2, \ldots,
$$

which corresponds to the control

$$
u(t)=-\dot{v}(t)=-\frac{3}{5} \frac{1}{(t-k \Delta)^{2 / 5}}, k \Delta<t \leqslant(k+1) \Delta, 0<\Delta \leqslant 1 .
$$

The maximum of the expression of Eq. (31) is attained at the time instant

$$
T(\Delta)=\left[\Delta^{-3 / 5}\right] \Delta+\left(1-\left[\Delta^{-3 / 5}\right] \Delta^{3 / 5}\right)^{5 / 3}
$$

and is defined by

$$
x(T)=k \Delta\left(1-\left(\frac{1}{8}+\frac{k}{2}\right) \Delta^{3 / 5}\right)+\frac{3}{8}\left(1-k \Delta^{3 / 5}\right)^{8 / 3}, k=\left[\Delta^{-3 / 5}\right] .
$$

The square brackets denote the integer part of the expression enclosed.

Hence, the control

$$
u(t)= \begin{cases}-\frac{3}{5} \frac{1}{(t-k \Delta)^{2 / 5}}, & k \Delta<t \leqslant(k+1) \Delta, t \leqslant T, \\ 0, & t>T\end{cases}
$$

is optimal, if the inequality of Eq. (16) holds for this control. We will prove this inequality.

Let $t_{2} \leqslant T$ and, hence, $t_{1}<T$. The function $v(t)$ for $t \leqslant T$ has the form of Eq. (32). According to the constraint $0<t_{2}-t_{1} \leqslant \Delta$, the instants $t_{1}$ and $t_{2}$ can belong to either one interval of continuity of the control of Eq. (36),

$$
k \Delta<t_{1} \leqslant(k+1) \Delta, k \Delta<t_{2} \leqslant(k+1) \Delta,
$$

or two adjacent intervals of continuity,

$$
k \Delta<t_{1} \leqslant(k+1) \Delta,(k+1) \Delta<t_{2} \leqslant(k+2) \Delta .
$$


If $t_{1}$ and $t_{2}$ belong to one interval of continuity of the control, then, in accordance with Eq. (32), we have

$$
v\left(t_{2}\right)-v\left(t_{1}\right)=\left(t_{2}-k \Delta\right)^{3 / 5}-\left(t_{1}-k \Delta\right)^{3 / 5} .
$$

The right-hand side of this relation satisfies the inequality

$$
\left(t_{2}-k \Delta\right)^{3 / 5}-\left(t_{1}-k \Delta\right)^{3 / 5} \leqslant\left(t_{2}-t_{1}\right)^{3 / 5} .
$$

This inequality is in fact the inequality of Eq. (23) in which $t_{1}$ and $t_{2}$ have been replaced by $\left(t_{1}-k \Delta\right)$ and $\left(t_{2}-k \Delta\right)$, respectively. Such a replacement is justified, since $t_{1}$ and $t_{2}$ in Eq. (23) can be treated as abstract variables that satisfy the conditions $t_{1}>0$ and $t_{2}>t_{1}$. The inequalities of Eqs (39) and (40) imply that of Eq. (16).

If $t_{1}$ and $t_{2}$ belong to two adjacent intervals of continuity of the control, then

$$
v\left(t_{2}\right)-v\left(t_{1}\right)=\Delta^{3 / 5}+\left(t_{2}-(k+1) \Delta\right)^{3 / 5}-\left(t_{1}-k \Delta\right)^{3 / 5}
$$

and Eq. (16) is equivalent to the inequality

$$
\begin{aligned}
& \Delta^{3 / 5}+\left(t_{2}-(k+1) \Delta\right)^{3 / 5}-\left(t_{1}-k \Delta\right)^{3 / 5}-\left(t_{2}-t_{1}\right)^{3 / 5} \leqslant 0, \\
& k \Delta<t_{1} \leqslant(k+1) \Delta,(k+1) \Delta<t_{2} \leqslant t_{1}+\Delta .
\end{aligned}
$$

We denote the left-hand side of Eq. (42) by $\chi\left(t_{2}\right)$ and will treat it as a function of the argument $t_{2}$ on the time interval $(k+1) \Delta<t_{2} \leqslant t_{1}+\Delta$. The straightforward substitution shows that $\chi\left(t_{1}+\Delta\right)=0$. Differentiating $\chi\left(t_{2}\right)$ yields

$$
\frac{d \chi}{d t_{2}}=\frac{3}{5}\left(t_{2}-(k+1) \Delta\right)^{-2 / 5}-\frac{3}{5}\left(t_{2}-t_{1}\right)^{-2 / 5} .
$$

From the inequality $t_{1} \leqslant(k+1) \Delta$ it follows that $t_{2}-(k+1) \Delta \leqslant t_{2}-t_{1}$. Hence, the minuend on the righthand side of Eq. (43) is greater than or equal to the subtrahend and, in addition, $d \chi\left(t_{2}\right) / d t_{2} \geqslant 0$ on the interval $(k+1) \Delta<t_{2} \leqslant t_{1}+\Delta$. Then, with reference to the relation $\chi\left(t_{1}+\Delta\right)=0$, we obtain $\chi\left(t_{2}\right) \leqslant 0$ on this time interval, which proves the inequality of Eq. (42) and, hence, that of Eq. (16).

Let now at least one of the instants $t_{1}$ or $t_{2}$ exceed $T$ of Eq. (34). In accordance with Eqs (12), (34), and (36), we have $v(t) \equiv 1$ for $t>T$. If $t_{1}>T$ (and, hence, $t_{2}>T$ ), then $v\left(t_{2}\right)-v\left(t_{1}\right)=0$ and the inequality of Eq. (16) apparently holds. Consider the case where $t_{1} \leqslant T$ but $t_{2}>T$. We have proved that $v\left(t_{2}\right)-v\left(t_{1}\right) \leqslant\left(t_{2}-t_{1}\right)^{3 / 5}$ for $0 \leqslant t_{1} \leqslant t_{2} \leqslant T$. Substitute $t_{2}=T$ into this inequality to obtain, with reference to the relation $v(T)=1$, that $1-v\left(t_{1}\right) \leqslant\left(T-t_{1}\right)^{3 / 5}$ and, hence, $1-v\left(t_{1}\right) \leqslant\left(t_{2}-t_{1}\right)^{3 / 5}$. Since $v(t) \equiv 1$ for $t \geqslant T$ for the control of Eq. (36), we have $v\left(t_{2}\right)-v\left(t_{1}\right) \leqslant\left(t_{2}-t_{1}\right)^{3 / 5}$ for $t_{1} \leqslant T$ and $t_{2}>T$.

This completes the proof of optimality of the control of Eq. (36) for $\Delta<1$.

We will represent the final solution of the optimal control problem. The optimal control $u_{0}(t)$ has the form

$$
\begin{aligned}
u_{0}(t) & =\left\{\begin{array}{ll}
-\frac{3}{5} \frac{1}{(t-k \Delta)^{2 / 5}}, & k \Delta<t \leqslant(k+1) \Delta, \\
0, & \text { if } t>T
\end{array}\right\} T, \\
T & =\left[\Delta^{-3 / 5}\right] \Delta+\left(1-\left[\Delta^{-3 / 5}\right] \Delta^{3 / 5}\right)^{5 / 3},
\end{aligned}
$$

if $\Delta<1$ or

$$
u_{0}(t)= \begin{cases}-\frac{3}{5} t^{-2 / 5}, & \text { if } t \leqslant 1, \\ 0, & \text { if } t>1,\end{cases}
$$

if $\Delta \geqslant 1$.

The minimum of the maximum displacement (deceleration distance) of the particle for the optimal control is defined by

$$
J_{1}\left(u_{0}\right)=k \Delta\left(1-\left(\frac{1}{8}+\frac{k}{2}\right) \Delta^{3 / 5}\right)+\frac{3}{8}\left(1-k \Delta^{3 / 5}\right)^{8 / 3}, k=\left[\Delta^{-3 / 5}\right],
$$

if $\Delta<1$ or

$$
J_{1}\left(u_{0}\right)=3 / 8
$$

if $\Delta \geqslant 1$.

The corresponding value of the HIC functional is equal to the maximum value allowed for this quantity, $J_{2}\left(u_{0}\right)=1$. 


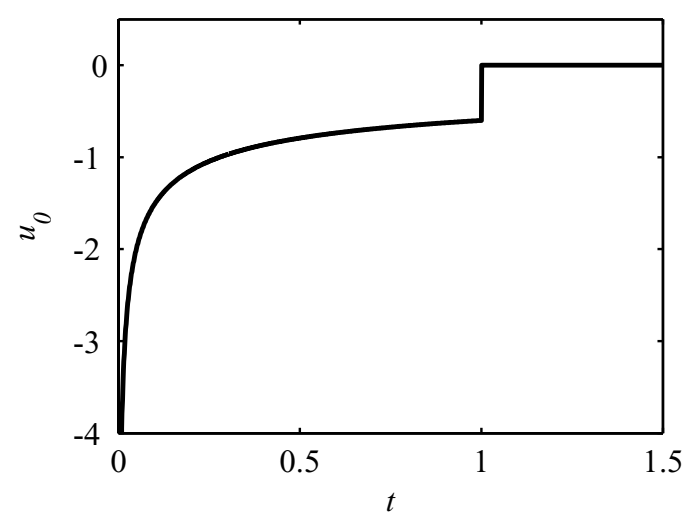

Fig. 2. Optimal control for $\Delta=1$.

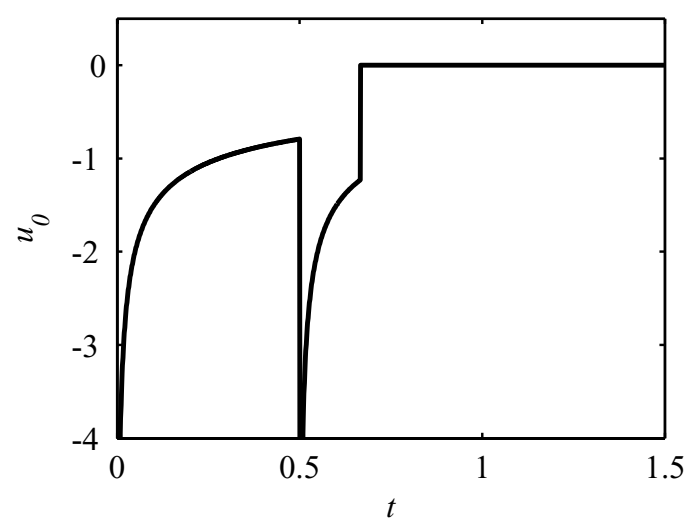

Fig. 3. Optimal control for $\Delta=0.5$.

\section{Analysis and discussion of the results}

We will list the characteristic qualitative features of the behavior of the optimal control of Eqs (44)-(45) and the minimum deceleration distance of Eqs (46)-(47), depending on the dimensionless parameter $\Delta$.

The optimal control $u_{0}(t)$ is constant in sign (negative) on the deceleration interval $(0, T]$, is continuous on the intervals $k \Delta<t<(k+1) \Delta$ and has discontinuities at the points $t=k \Delta, k=0,1, \ldots,\left[\Delta^{-3 / 5}\right]$. (The square brackets denote the integer part of the corresponding number.) The function $u_{0}(t)$ monotonically increases on the intervals of continuity and tends to $-\infty$ on the right at the points of discontinuity. The points of discontinuity occur with period $\Delta$, beginning from $t=0$. The number of the discontinuity points on the deceleration interval is $\left[\Delta^{-3 / 5}\right]+1$. This number increases without limit as $\Delta \rightarrow 0$.

Figures 2-4 show the time history of the optimal control on the interval $(0, T]$ for $\Delta=1$ (Fig. 2), $\Delta=0.5$ (Fig. 3), and $\Delta=0.25$ (Fig. 4).

Consider the relation of Eq. (11) that expresses the dimensionless parameter $\Delta$ (denoted by $\Delta^{\prime}$ in Eq. (11)) in terms of the primary dimensional parameters $\Delta, H$, and $v_{0}$. The lower the $H$ and/or the greater the $v_{0}$, the lower the $\Delta^{\prime}$. Therefore, a decrease in the maximum allowable value of the HIC and/or increase in the initial velocity can lead to the appearance of additional points of discontinuity in the optimal control law at which the magnitude of the control force becomes infinite.

These observations are illustrated by a diagram in Fig. 5. This diagram shows the curves of constant levels of the minimal deceleration distance $J_{1}\left(u_{0}\right)$ as a function of the impact velocity $v_{0}$ and the maximum tolerable value of the HIC functional, $H$. The numbers labeling the curves correspond to the values of $J_{1}\left(u_{0}\right)$ measured in meters. The quantity $v_{0}$ is measured in kilometers per hour and $H$ in standard units adopted in engineering biomechanics. These 


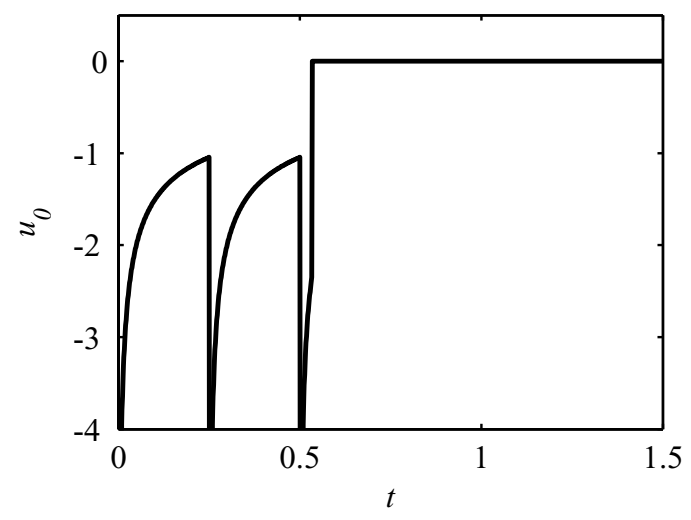

Fig. 4. Optimal control for $\Delta=0.25$.

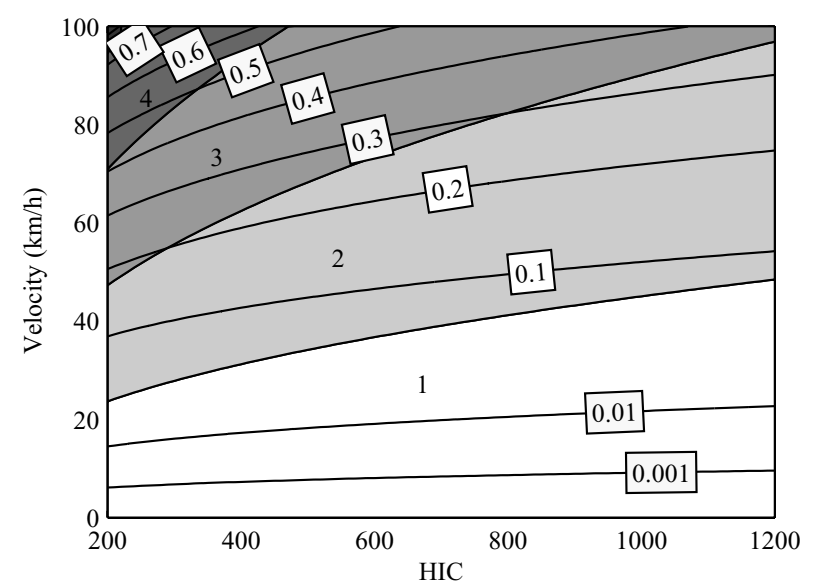

Fig. 5. Curves of constant levels of $J_{1}\left(u_{0}\right)$ as a function of $v_{0}$ and $H$. Regions with different numbers of discontinuity points of the optimal control.

units imply that $a$ in Eq. (1) is measured in units of $g$ (the acceleration due to gravity), i.e., the definition of Eq. (1) is replaced by

$$
\mathrm{HIC}=\max _{t_{1}, t_{2}, t_{2}-t_{1} \leqslant \Delta}\left\{\left[\frac{1}{t_{2}-t_{1}} \int_{t_{1}}^{t_{2}} \frac{a(t)}{g} d t\right]^{2.5}\left(t_{2}-t_{1}\right)\right\},
$$

and time is measured in seconds. The diagram of Fig. 5 corresponds to $\Delta=15 \mathrm{~ms}$.

There are several regions on the diagram corresponding to different number of discontinuity points of the optimal control. The number of the region indicates the number of the discontinuity points.

In accordance with Eqs (46) and (47), the minimum (dimensionless) deceleration distance $J_{1}\left(u_{0}\right)$ monotonically increases from 0 to $3 / 8$, as the (dimensionless) parameter $\Delta$ increases from 0 to 1 . As $\Delta$ continues to increase beyond 1 , the value $J_{1}\left(u_{0}\right)$ remains equal to $3 / 8$. The plot of $J_{1}\left(u_{0}\right)$ versus $\Delta$ on the interval $0 \leqslant \Delta<1$ is shown in Fig. 6 . The asymptotic behavior of this curve for small $\Delta$ is determined by the relation

$$
J_{1}\left(u_{0}\right) \sim \frac{1}{2} \Delta^{2 / 5}, \Delta \rightarrow 0 .
$$

For applications, it is convenient to have an explicit relationship between the minimum value of the performance index and the input parameters of the problem represented in the primary dimensional variables. To express the relations 


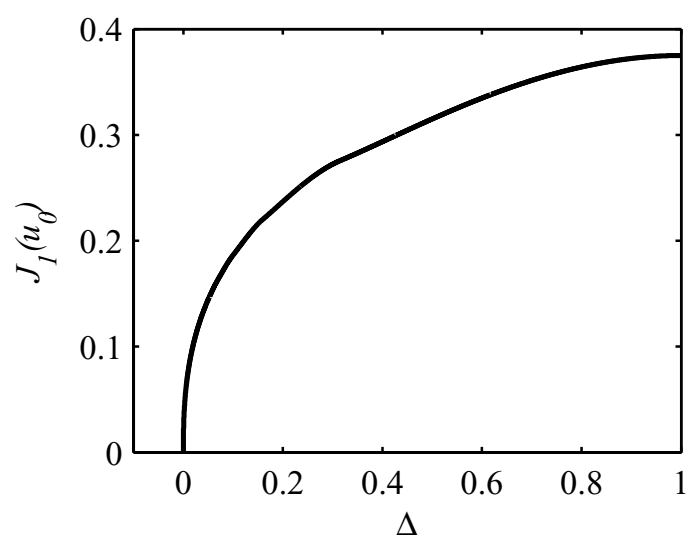

Fig. 6. $J_{1}\left(u_{0}\right)$ as a function of $\Delta$.

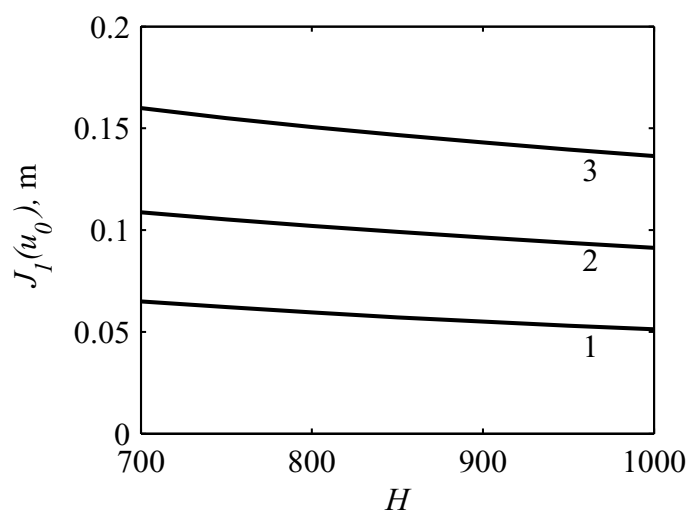

Fig. 7. $J_{1}\left(u_{0}\right)$ as a function of $H$ for various $v_{0}$ : Curve $1-v_{0}=40 \mathrm{~km} / \mathrm{h}$; curve $2-v_{0}=50 \mathrm{~km} / \mathrm{h} ;$ curve $3-v_{0}=60 \mathrm{~km} / \mathrm{h}$.

of Eqs (46) and (47) in the dimensional variables, multiply the right-hand sides of the cited relations by $v_{0}^{8 / 3} / H^{2 / 3}$ and change $\Delta$ to $H^{2 / 3} \Delta / v_{0}^{5 / 3}$ in accordance with Eq. (11). This representation shows that the minimum deceleration distance in the optimal control problem monotonically increases as the initial velocity, $v_{0}$, increases and monotonically decreases as the maximum value allowed for the HIC functional, $H$, increases. Figure 7 shows $J_{1}\left(u_{0}\right)$ as a function of $H$ in the dimensional variables for $\Delta=0.015 \mathrm{~s}$ and $v_{0}=40$ (curve 1), 50 (curve 2) 60 (curve 3) km/h.

For $H^{2 / 3} \Delta / v_{0}^{5 / 3} \geqslant 1$ (which corresponds to $\Delta \geqslant 1$ in the dimensionless variables) and $H^{2 / 3} \Delta / v_{0}^{5 / 3} \ll 1$ ( $\Delta \ll 1$ in the dimensionless variables), this dependence can be represented by simple analytical expressions. Proceeding to the dimensional variables in Eqs (47) and (49), we obtain

$$
\begin{aligned}
& J_{1}\left(u_{0}\right)=\frac{3}{8} \frac{v_{0}^{8 / 3}}{H^{2 / 3}}, \text { if } \Delta \frac{H^{2 / 3}}{v_{0}^{5 / 3}} \geqslant 1 . \\
& J_{1}\left(u_{0}\right) \sim \frac{v_{0}^{2}}{2 H^{2 / 5}} \Delta^{2 / 5}, \text { if } \Delta \frac{H^{2 / 3}}{v_{0}^{5 / 3}} \ll 1 .
\end{aligned}
$$

The region of Eq. (50) is important for applications, since it covers a rather wide range of velocities for the values of $\Delta$ and $H$ adopted by car crash test standards. The region of Eq. (51) is of interest, apparently, only from the viewpoint of theory. The corresponding asymptotic relation shows the behavior of the optimal value of $J_{1}$ as $v_{0}$ increases without limit or $H$ tends to zero. 
The dimensionless deceleration time $T$ corresponding to the optimal control of Eq. (44) monotonically increases from 0 to 1 as $\Delta$ increases from 0 to 1 . For $\Delta>1$, the deceleration time corresponding to the control of Eq. (45) is equal to unity. The behavior of the function $T(\Delta)$ for small $\Delta$ is defined by the asymptotic relation

$$
T \sim \Delta^{2 / 5}, \Delta \rightarrow 0
$$

To proceed to the primary dimensional variables in the expressions for $T$, one should, in accordance with Eq. (11), multiply the corresponding expressions by $v_{0}^{5 / 3} / H^{2 / 3}$ and change $\Delta$ to $H^{2 / 3} \Delta / v_{0}^{5 / 3}$. In the dimensional variables, $T$ decreases as $H$ increases and/or $v_{0}$ decreases. We choose to present the expressions for $T$ for $H^{2 / 3} \Delta / v_{0}^{5 / 3} \geqslant 1$ (which corresponds to $\Delta \geqslant 1$ in the dimensionless variables) and $H^{2 / 3} \Delta / v_{0}^{5 / 3} \ll 1(\Delta \ll 1$ in the dimensionless variables),

$$
\begin{aligned}
& T=\frac{v_{0}^{5 / 3}}{H^{2 / 3}}, \text { if } \Delta \frac{H^{2 / 3}}{v_{0}^{5 / 3}} \geqslant 1 . \\
& T \sim \frac{v_{0}}{H^{2 / 5}} \Delta^{2 / 5}, \text { if } \Delta \frac{H^{2 / 3}}{v_{0}^{5 / 3}} \ll 1 .
\end{aligned}
$$

As was the case for the expressions of Eqs (50) and (51) for the optimal deceleration distance, the expression of Eq. (53) for the deceleration time is important for applications, since it corresponds to a broad range of the parameters typical of car crash test conditions, whereas the expression of Eq. (54), which characterizes the increase in the deceleration time as the initial velocity, $v_{0}$, increases without limit or $H$ tends to zero, can be of interest only for theory.

\section{Minimization of the HIC for constrained deceleration distance}

Along with Problem 1, considered thus far, it is reasonable to consider the problem in which the HIC is the performance index to be minimized and the deceleration distance is subjected to a constraint.

Problem 2. For the system governed by the differential equation of (6) subjected to the initial conditions of Eq. (7), find an optimal control $u=u^{0}(t)$ in the class of integrable functions to minimize the criterion $J_{2}$, provided that the peak displacement, $J_{1}$, does not exceed a prescribed positive number $D$, i.e.,

$$
J_{2}\left(u^{0}\right)=\min _{u}\left\{J_{2}(u) \mid J_{1}(u) \leqslant D\right\}
$$

In terms of the analysis of the limiting capabilities of the prevention of impact-induced head injuries by means of impact isolation coatings, Problem 2 corresponds to the maximum reduction of the expected severity of the head injury in the case where the maximum thickness allowed for the coating is restricted.

Problem 2 is dual to Problem 1 in the sense that knowing the solution of Problem 1 as a function of $H$, one can obtain the solution of Problem 2. (The values of $v_{0}$ and $\Delta$ are fixed the same for both problems.) For the optimal controls of Problems 1 and 2 we assign the indices $H$ and $D$, respectively, to indicate the dependence of these controls on the maximum value allowed for the constrained criterion, i.e., instead of $u_{0}$ and $u^{0}$ we will write $u_{0}^{H}$ and $u_{D}^{0}$. Let $f(H)$ denote the minimum deceleration distance in Problem 1 as a function of the maximum value allowed for the HIC, i.e., $f(H)=J_{1}\left(u_{0}^{H}\right)$. From the solution of Problem 1 it follows that the function $f(H)$ is defined for all positive $H$, is continuous, and monotonically decreases from $+\infty$ to 0 as $H$ increases from 0 to $+\infty$. Hence, this function has the inverse $f^{-1}(D)$, which is defined on the half-line $0<D<\infty$, is continuous, and monotonically decreases from $+\infty$ to 0 . In this case, as shown, for example, in [1] or [2], the solutions of Problems 1 and 2 are related by

$$
u_{D}^{0}(t)=u_{0}^{f^{-1}(D)}, J_{2}\left(u_{D}^{0}\right)=f^{-1}(D) .
$$




\section{Alternative control laws}

In this section, we consider two "reasonable" deceleration laws - the constant force deceleration law and the power law of braking - and compare the values of the criterion $J_{1}$ of Eq. (8) provided by these control laws with the absolute minimum provided by the optimal control.

\subsection{Constant force deceleration}

This deceleration law is defined by

$$
u=\left\{\begin{array}{l}
-w, \text { if } 0 \leqslant t \leqslant t^{*}=w^{-1}, \\
0, \quad \text { if } t>t^{*}
\end{array},\right.
$$

where $w$ is the positive constant to be determined so as to minimize the functional $J_{1}$ under the constraint $J_{2} \leqslant 1$. One can say that this minimization gives the solution of Problem 1 in the one-parameter (with the parameter $w$ ) class of functions of Eq. (57).

When subjected to the control of Eq. (57), the object (a particle) uniformly decelerates from the initial velocity $v_{0}=1$ to a complete stop at the time instant $t^{*}=w^{-1}$ and then remains in the position $x=x\left(t^{*}\right)=1 /(2 w)$. Thus,

$$
J_{1}=x\left(t^{*}\right)=1 /(2 w) \text {. }
$$

Substitute Eq. (57) into the expression of Eq. (9) for the functional $J_{2}$ to obtain

$$
J_{2}=\left\{\begin{array}{ll}
w^{5 / 2} \Delta, & \text { if } \Delta \leqslant w^{-1} \\
w^{3 / 2}, & \text { if } \Delta>w^{-1}
\end{array} .\right.
$$

The minimization of the function $J_{1}$ of Eq. (58) under the constraint $J_{2} \leqslant 1$, where $J_{2}$ is defined by the expressions of Eq. (59), yields

$$
w= \begin{cases}\Delta^{-2 / 5}, & \text { if } \Delta \leqslant 1 \\ 1, & \text { if } \Delta>1\end{cases}
$$

and, accordingly,

$$
J_{1}=\left\{\begin{array}{ll}
\Delta^{2 / 5} / 2, & \text { if } \Delta \leqslant 1 \\
1 / 2, & \text { if } \Delta>1
\end{array} .\right.
$$

\subsection{Power-law deceleration force}

This law has the form

$$
u=\left\{\begin{array}{l}
-\frac{A}{t^{\beta}}, \text { if } 0 \leqslant t \leqslant t_{*}=\left(\frac{1-\beta}{A}\right)^{\frac{1}{1-\beta}}, \\
0, \quad \text { if } t>t_{*}
\end{array}\right.
$$

where $A$ and $\beta$ are parameters, $A>0$ and $\beta<1$ (for $\beta \geqslant 1$ the function of Eq. (62) has a non-integrable singularity at the point $t=0$ ). When subjected to the control of Eq. (62), the object decelerates from the initial velocity $v_{0}=1$ to a complete stop at the time instant $t_{*}$ in the position

$$
J_{1}=x\left(t_{*}\right)=\frac{(1-\beta)^{\frac{2-\beta}{1-\beta}}}{A^{\frac{1}{1-\beta}}(2-\beta)} .
$$

The parameters $A$ and $\beta$ are to be found so as to minimize the quantity of $J_{1}$ of Eq. (63) under the constraint $J_{2} \leqslant 1$.

Substituting the control of Eq. (62) into the right-hand side of Eq. (9) and calculating the maximum of the expression in the curly brackets with respect to $t_{1}$ and $t_{2}\left(0 \leqslant t_{2}-t_{1} \leqslant \Delta\right)$, we obtain 


$$
J_{2}= \begin{cases}\left(\frac{A}{1-\beta}\right)^{2.5} \Delta^{1-2.5 \beta}, & \text { if } \Delta \leqslant\left(\frac{1-\beta}{A}\right)^{\frac{1}{1-\beta}} \\ \left(\frac{A}{1-\beta}\right)^{\frac{1.5}{1-\beta}}, & \text { if } \Delta>\left(\frac{1-\beta}{A}\right)^{\frac{1}{1-\beta}}\end{cases}
$$

for

$$
\beta \leqslant 2 / 5 \text {. }
$$

It can be shown that $J_{2}=\infty$ for $\beta>2 / 5$.

We wish to find the minimum of the function $J_{1}$ of Eq. (63) with respect to $A$ and $\beta$ under the constraints $A \geqslant 0$, $\beta \leqslant 2 / 5$, and $J_{2} \leqslant 1$, where $J_{2}$ is defined by Eq. (64). First, we will calculate the minimum with respect to $A$ for fixed $\beta$. Since $J_{1}$ decreases, whereas $J_{2}$ increases in $A$, the desired minimum is attained at $J_{2}=1$. In accordance with Eq. (64), we have

$$
\begin{aligned}
& A=\left\{\begin{array}{ll}
(1-\beta) \Delta^{\frac{2.5 \beta-1}{2.5}}, & \text { if } \Delta<1 \\
A=1-\beta, & \text { if } \Delta \geqslant 1
\end{array},\right. \\
& J_{1}=\left\{\begin{array}{ll}
\frac{1-\beta}{2-\beta} \Delta^{\frac{1-2.5 \beta}{2.5(1-\beta)}}, & \text { if } \Delta<1 \\
\frac{1-\beta}{2-\beta}, & \text { if } \Delta \geqslant 1
\end{array} .\right.
\end{aligned}
$$

Minimize then the function of Eq. (67) under the constraint of Eq. (65) to obtain the final solution

$$
\begin{aligned}
& J_{1}= \begin{cases}\frac{1}{2} \Delta^{2 / 5}, & \text { if } \Delta<e^{-5 / 6} \\
-\frac{3 \ln \Delta}{5} \Delta^{\frac{5+8 \ln \Delta}{5 \ln \Delta}}, & \text { if } e^{-5 / 6} \leqslant \Delta \leqslant e^{-5 / 8}, \\
\frac{3}{8}, & \text { if } \Delta>e^{-5 / 8}\end{cases} \\
& \beta= \begin{cases}0, & \text { if } \Delta<e^{-5 / 6} \\
\frac{5+6 \ln \Delta}{5+3 \ln \Delta}, & \text { if } e^{-5 / 6} \leqslant \Delta \leqslant e^{-5 / 8} \\
\frac{2}{5}, & \text { if } \Delta>e^{-5 / 8}\end{cases} \\
& A= \begin{cases}\Delta^{-2 / 5}, & \text { if } \Delta<e^{-5 / 6} \\
-\frac{3 \ln \Delta}{5+3 \ln \Delta} \Delta^{\frac{15+24 \ln \Delta}{5(5+3 \ln \Delta)}}, & \text { if } e^{-5 / 6} \leqslant \Delta \leqslant e^{-5 / 8} \\
\frac{3}{5}, & \text { if } \Delta>e^{-5 / 8}\end{cases}
\end{aligned}
$$

From Eqs (62), (69), and (70) it follows that for $\Delta>e^{-5 / 8}$, the control of Eq. (62) with the optimal parameters $A$ and $\beta$ coincides in form with the control of Eq. (45), which is optimal for $\Delta \geqslant 1$. For $\Delta<e^{-5 / 6}$, the control of Eq. (62) with the parameters of Eqs (69) and (70) coincides with the constant force control of Eq. (60).

From Eqs (49), (61), and (68) it follows that for small $\Delta$, the optimal control of Eq. (44), the constant force control of Eq. (60), and the control of Eq. (62) with the parameters of Eqs (69) and (70) are asymptotically equivalent, since these control laws provide the same asymptotic behavior for the performance index $J_{1}$ as $\Delta \rightarrow 0$.

Calculate the measure of non-optimality of the control of Eqs (57) and (60) and that of Eqs (62), (69), and (70) with respect to the functional $J_{1}$, defined by

$$
\eta_{(57)}=\frac{J_{1}^{(57)}-J_{1}\left(u_{0}\right)}{J_{1}\left(u_{0}\right)}, \eta_{(62)}=\frac{J_{1}^{(62)}-J_{1}\left(u_{0}\right)}{J_{1}\left(u_{0}\right)},
$$




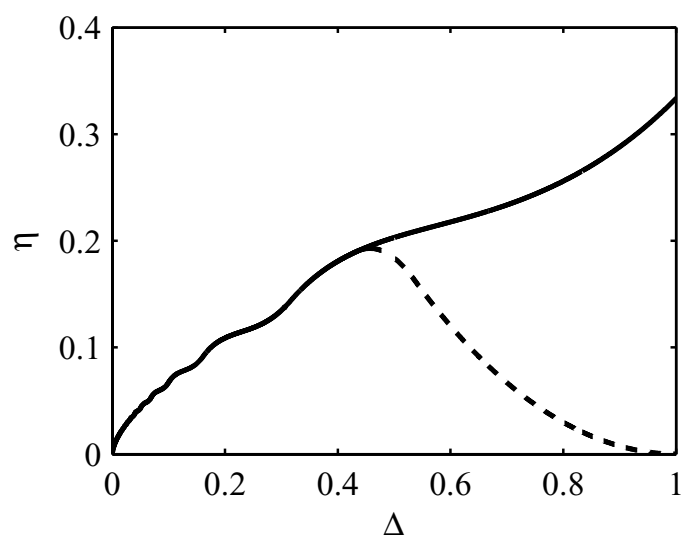

Fig. 8. Non-optimality measure for the constant-force and power-law controls.

where $J_{1}^{(57)}$ denotes the function of Eq. (61), corresponding to the control of Eq. (57) with w of Eq. (60), $J_{1}^{(62)}$ denotes the function of Eq. (68), corresponding to the control of Eq. (62) with the parameters of Eqs (69) and (70), and $J_{1}\left(u_{0}\right)$ denotes the function of Eqs (46) and (47) that determines the minimum deceleration distance of the object for the optimal control of Eqs (44) and (45).

The definitions of these functions imply that

$$
\eta_{(57)}=1 / 3, \eta_{(62)}=0, \text { if } \Delta \geqslant 1 .
$$

The graphs of the functions $\eta_{(57)}(\Delta)$ (solid curve) and $\eta_{(62)}(\Delta)$ (dashed line) for $\Delta<1$ are plotted in Fig. 8.

\section{Conclusion}

The HIC is an empirical integral functional that evaluates the expected severity of the impact-induced head injury of a human being as a function of the magnitude of the acceleration of the brain center of mass and the duration of the impact pulse. In some countries (in particular, in the USA), the HIC is utilized as a standard crashworthiness criterion for automobiles and as the basic performance index of coatings of sporting and children playgrounds and impact isolation helmets. Automobile crash tests, as well as testing of playgrounds and helmets in which the HIC functional is calculated, involve human dummies with accelerometers integrated into the head. An important engineering problem associated with the improvement of crashworthiness indices of vehicles and sporting equipment with respect to the HIC is that of the design of impact isolation coatings or paddings (in helmets) which would provide the required degree of protection from head injuries and be as thin as possible. The limiting performance analysis of such coatings leads to an optimal control problem in which the functional to be minimized is the distance of deceleration of the head in contacting the coating, while the HIC functional is constrained. The solution of this problem allows one to determine the theoretical minimum of the coating thickness, as well as the optimal law of the head deceleration. In the present paper, an analytical solution of the optimal control problem has been constructed for a single-degree-of-freedom system that models the normal impact of the head against a surface, provided that the head is moving translationally. It is shown that the optimal deceleration law involves intervals on which the head acceleration magnitude is very large and tends to infinity at isolated points. The number of such points increases as the impact velocity increases and/or the maximum value allowed for HIC decreases.

\section{Acknowledgments}

This research was partly supported by NSF (grant BES-0302337), NATO (grant CBP.NR.NRCLG 982082), and the Russian Foundation for Basic Research (grants 05-01-00563 and 04-01-00222). 


\section{References}

[1] D.V. Balandin, N.N. Bolotnik and W.D. Pilkey, Optimal Protection from Impact, Shock, and Vibration, Gordon and Breach Science Publishers, Amsterdam, 2001.

[2] N.N. Bolotnik, Optimization of Shock and Vibration Isolation Systems (in Russian), Nauka, Moscow, 1983.

[3] Z.Q. Cheng, W.D. Pilkey, J.R. Crandall, C.R. Bass and K. Darvish, Limiting performance of helmets for the prevention of head injury, Shock and vibration 6(5,6) (1999), 299-320.

[4] C.W. Gadd, Use of a weighted-impulse criterion for estimating injury hazard, in: Proceedings of the 10th Stapp Car Crash Conference, SAE Paper No. 660793, Society of Automotive Engineers, 1966.

[5] E.S. Gurdjian, V.R. Hodgsom, W.G. Hardy, L.M. Patrick and H.R. Lissner, Evaluation of the protective characteristics of helmets in sports, Journal of Trauma 4 (1964), 273-286.

[6] E.S. Gurdjian, H.R. Lissner, F.R. Latimer, B.F. Haddad and J.E. Webster, Quantitative determination of acceleration and intercranial pressure in experimental head injury, Neurology 3 (1953), 417-423.

[7] E.S. Gurdjian, V.L. Roberts and L.M. Thomas, Tolerance curve of acceleration and intercranial pressure and protective index in experimental head injury, Journal of Trauma 6 (1966), 600-604.

[8] J. Hutchinson, M.J. Kaiser and M.H. Lankarani, The head injury criterion (HIC) functional, Applied Mathematics and Computation 96 (1998), 1-16.

[9] F.J. Lockett, Biomechanics justification of empirical head tolerance criteria, Journal of Biomechanics 18 (1985), 217-245.

[10] J.A. Newman, Head injury criteria in automotive crash testing, in: Proceedings of the 24th Stapp Car Crash Conference, SAE Paper No. 801317, Society of Automotive Engineers, 1980.

[11] Y. Okamoto, A. Akiyama, K. Nagatomi and T. Tsuruga, Concept of hood design for possible reduction in pedestrian head injury, in: Proceedings of the 14th International Technical Conference on the Enchanced Safety of Vehicles, Munich, 1994.

[12] M.R. Shorten and J.A. Himmelsbach, Shock attenuation of sports surfaces, in: The Engineering of Sport IV: Proceedings of the 4th International Conference on the Engineering of Sport, S. Ujihashi and S.J. Haake, eds, Oxford, Blackwell Science, 2002.

[13] J. Versace, A review of the severity index, in: Proceedings of the 15th Stapp Car Crash Conference, SAE Paper No. 710881, Society of Automotive Engineers, 1971. 

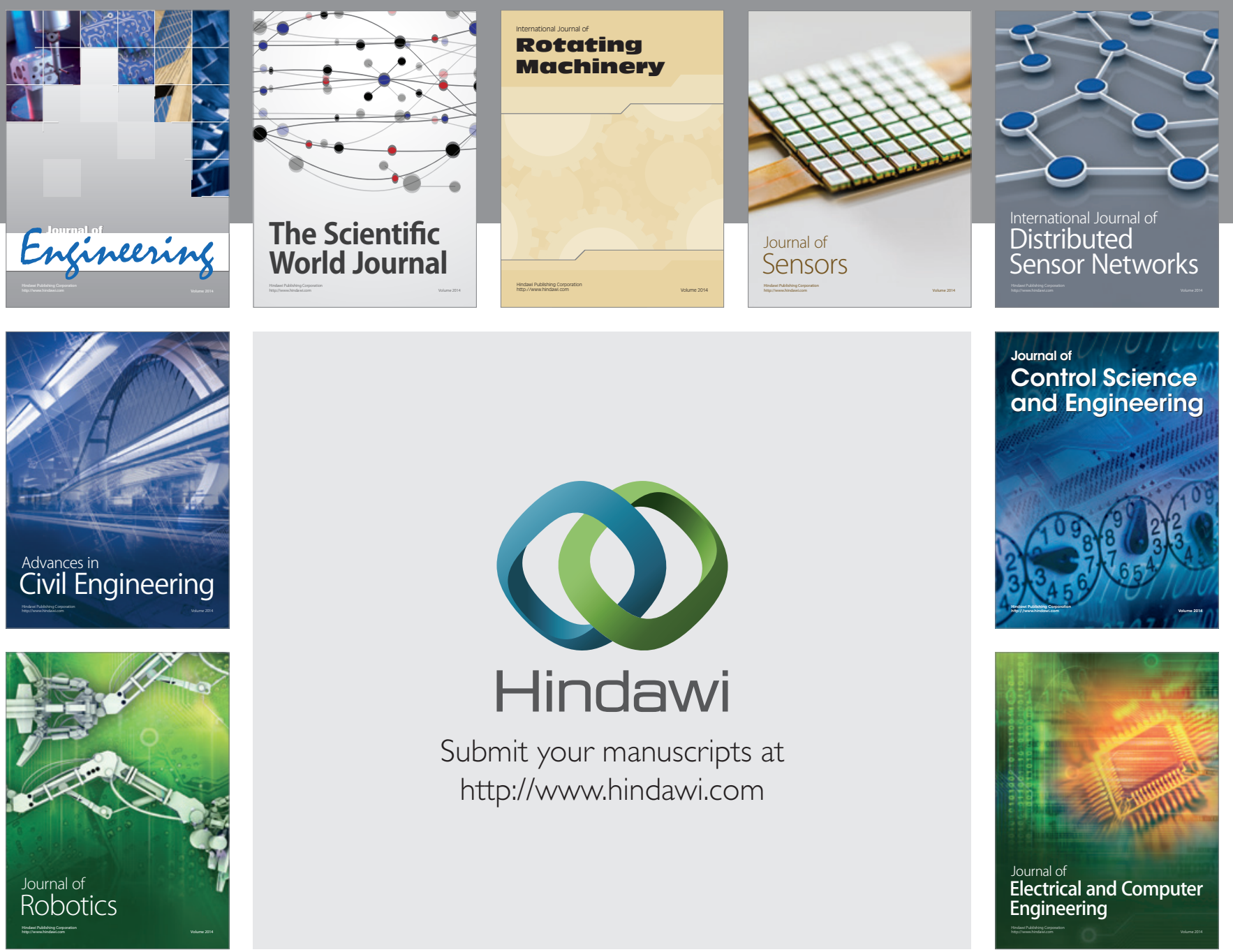

Submit your manuscripts at

http://www.hindawi.com
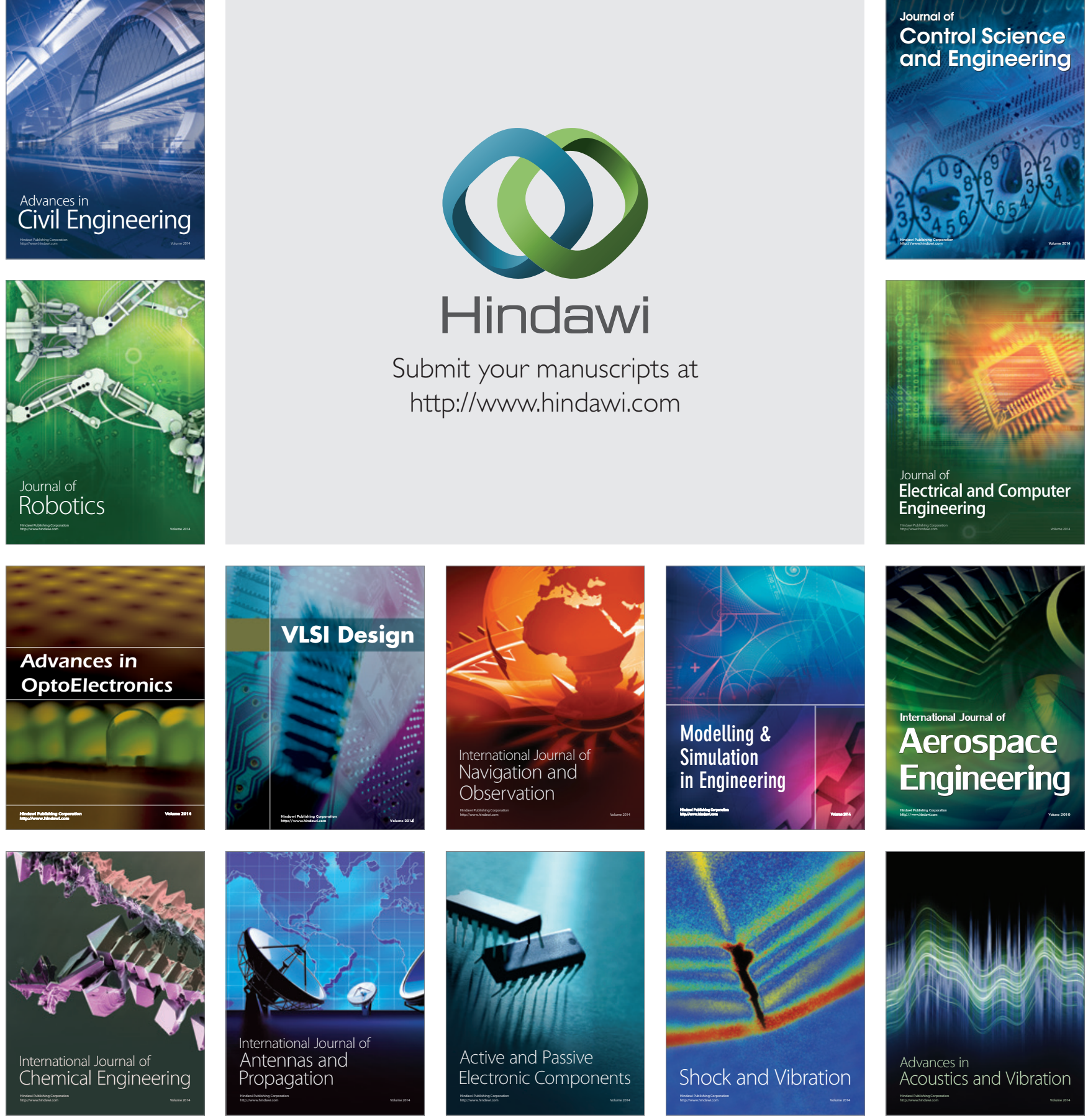